\title{
Recuperación de iones cobre por flotación con amilxantato de potasio(*)
}

\author{
B. Ramírez-Serrano*, A.L. Coello-Velázquez*, A. Bernardo**, E. Afif** y J.M. Menéndez-Aguado**
}

\begin{abstract}
Resumen Se realiza un estudio sobre la flotación en columnas de iones cobre utilizando como reactivo colector amilxantato de potasio. Se determina experimentalmente el efecto de la modificación de las variables relación colector/metal, velocidad superficial del gas y del líquido sobre la recuperación, a partir del análisis del modelo matemático-estadístico que caracteriza la flotación de cobre, así como de los fenómenos físico-químicos que tienen lugar, donde se comprueba el papel preponderante de la variable relación colector/metal en la recuperación de cobre. Se estudia además, el efecto del $\mathrm{pH}$ como elemento determinante de las características químicas del sistema en la recuperación y la cinética del proceso de flotación. Los resultados experimentales muestran que es posible la recuperación del cobre disuelto para el rango de $\mathrm{pH}$ de 4,5 a 12 con predominio de la flotación de precipitados.
\end{abstract}

Palabras clave Amilxantato de potasio; Complejos de cobre; Columna de flotación; Flotación de precipitados.

\section{Recovery of copper ion by flotation with potassium amylxanthate}

\begin{abstract}
In this paper a study about the column flotation process of copper ion employing potassium amylxanthate as collector reagent is carried out. The effect on the recovery of copper ion by the modification of variables such as collector/metal relation and surface velocity of gas and liquid was determined experimentally by the analysis of the statistic-mathematical model of the copper flotation process, as well as the physico-chemical phenomena that take place, showing the effect of the collector/metal relation in the process. The effect of $\mathrm{pH}$ as the main properties of the chemical system in the recovery and the kinetic of the flotation process is made too. The experimental results shows that the recovery of copper in the $\mathrm{pH}$ range of 4,5-12 is possible with prevalence of precipitate flotation.
\end{abstract}

Keywords

Amylxanthate; Copper complex; Column flotation; Precipitate flotation.

\section{INTRODUCCIÓN}

Los metales pesados normalmente son tóxicos y peligrosos, su acumulación en el organismo constituye un serio riesgo para la salud. Según Babel y Kurniawan ${ }^{[1]}$ el cobre se encuentra entre los elementos que provocan mayor riesgo para la salud.

Con frecuencia, el cobre se encuentra en soluciones residuales de diferentes procesos industriales y en los efluentes de las aguas de drenaje de minas. Para su tratamiento se han aplicado diferentes técnicas, tales como la precipitación, extracción con solventes orgánicos, electrólisis e intercambio iónico ${ }^{[2}$ y 3$]$. No obstante, cuando la concentración del metal se encuentra por debajo de $1.000 \mathrm{mg} / \mathrm{l}$ y la cantidad de efluente es elevada, la eficiencia de estos procesos disminuye $\mathrm{e}^{[4}$ y 5$]$.
De acuerdo con las investigaciones sobre el tratamiento de soluciones residuales contaminadas con cobre y otros metales ${ }^{[6-10]}$, la técnica de flotación ha demostrado su potencialidad no solo por la eficiencia del proceso, sino porque éstos se caracterizan por bajos costos de instalación, operación y consumo energético, fácil automatización, así como el tratamiento de grandes volúmenes de soluciones diluidas en poco tiempo ${ }^{[7,11-14]}$.

El uso de reactivos colectores xantogenados, ampliamente utilizados en la flotación de minerales de sulfurosos de cobre ${ }^{[15]}$, constituyen un elemento a tener en consideración dada su alta reactividad frente a los iones metálicos ${ }^{[16]}$ además de permitir el control independiente de la acción colectora y espumante ${ }^{[17]}$. El etilxantato y el dietilxantato de

(•) Trabajo recibido el día 23 de Octubre de 2011 y aceptado en su forma final el día 13 de febrero de 2012.

* Instituto Superior Minero Metalúrgico. Las Coloradas s/n C.P. 83329 Moa, Cuba.

** Escuela Politécnica de Mieres. Universidad de Oviedo. 
sodio han sido aplicados en la flotación iónica por aire disuelto de iones cobre(II), zinc y arsénico(V), a partir de soluciones sintéticas de iones individuales y sus mezclas; la eliminación de cobre en el rango de $\mathrm{pH}$ entre 2,5 y 5,5 no se afecta, pero requiere de un exceso de reactivo colector de un $10 \%$ [12 y 18$]$. Sin embargo, para $\mathrm{pH}$ inferior a 4,7 se verifica la descomposición parcial del etilxantato $^{[16,19-21]}$.

El dietilxantato de sodio, a pesar de exhibir propiedades similares y mostrar buenos resultados en la colección de cobre y zinc, tiene una utilización como colector limitada por el costo que representa su obtención $^{[22]}$.

Lazaridis et al. ${ }^{[23]}$ también reportan la aplicación de flotación iónica por aire disuelto con etilxantato en sistemas de cobre, hierro y níquel, de forma independiente y en sus mezclas. Según los autores, si el $\mathrm{pH}$ del sistema es igual a 2, la recuperación de cobre y hierro se reduce hasta $50 \%$ y $25 \%$ respectivamente, dependiendo de la concentración inicial de cobre. Es posible que el resultado se encuentre asociado con la descomposición del etilxantato; su tiempo de vida media a $\mathrm{pH} 2,5$ es de $120 \mathrm{~s}{ }^{\text {[24] }}$.

En la flotación, la solubilidad de las especies que se separan juega un rol fundamental, su combinación con las características de la dispersión y los parámetros de operación de la columna determina la efectividad del proceso ${ }^{[6}$ y 25].

En el caso de los xantatos, el incremento de la longitud de la cadena de carbono da lugar a la formación de compuestos con menor solubilidad ${ }^{[16]}$ como consecuencia del aumento de la fuerza de enlace del grupo aniónico y el catión metálico ${ }^{[26]}$, además de influir en su cinética de descomposición. En medio ácido, la cinética de descomposición del etilxantato es más rápida que la del amilxantato ${ }^{[27]}$.

El objetivo de este artículo es evaluar la remoción de los iones de cobre por flotación con amilxantato de potasio.

\section{METODOLOGÍA EXPERIMENTAL}

Los experimentos se llevaron a cabo en una columna de flotación a escala piloto, construida de metacrilato transparente de $0,10 \mathrm{~m}$ de diámetro x $5,5 \mathrm{~m}$ de altura. La figura 1 muestra la representación esquemática de la instalación, compuesta además por dos bombas peristálticas en las corrientes de alimentación y en colas, un manómetro diferencial, caudalímetros de líquido y gas y un compresor que garantiza una presión constante del gas en la alimentación de la columna.

El proceso de acondicionamiento de la solución se realiza en un recipiente con agitación constante.

Para el desarrollo de la investigación se lleva a cabo un diseño de experimentos del tipo factorial completo $\left(3^{3}\right)$, incluyendo dos réplicas por cada ensayo, lo que supuso un total de 81 ensayos. El diseño permite evaluar la influencia de las variables velocidad superficial del líquido, velocidad superficial del gas y la relación colector: metal en la recuperación de cobre y definir las mejores condiciones para llevar a cabo el proceso. Estas variables determinan en gran medida las características de la dis-

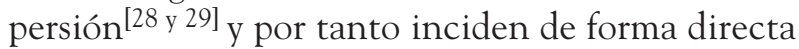
en la recuperación y cinética del proceso ${ }^{[6]}$; los valores de los niveles de las variables a estudiar se relacionan en la tabla I.

La selección de los niveles de la variable relación colector: metal se fundamenta en el estudio de las interacciones entre el cobre y el amilxantato ${ }^{[30]}$, el cual indica la formación de los complejos de cobre para todas las relaciones estudiadas. Se selecciona como nivel básico la relación colector: metal (amilxantato: cobre) 2:1 que se corresponde con la relación estequiométrica para la formación del complejo de cobre(II). En el caso de los niveles de la velocidad superficial del líquido y velocidad superficial del gas se tuvieron en cuenta otras experiencias ${ }^{[31-35]}$ desarrolladas en instalaciones similares. Previamente se

Tabla I. Niveles de estudio de las variables independientes

Table I. Independent variables level

\begin{tabular}{lccc}
\hline \multirow{4}{*}{ Niveles } & \multicolumn{3}{c}{ Variables independientes } \\
\cline { 2 - 4 } & $\begin{array}{c}\text { Velocidad superficial } \\
\text { del líquido Jl, cm/s }\end{array}$ & $\begin{array}{c}\text { Velocidad superficial } \\
\text { del gas Jg, cm/s }\end{array}$ & $\begin{array}{c}\text { Relación Colector: } \\
\text { Me R(C:Me) }\end{array}$ \\
\hline Mínimo (-1) & 0,4 & 0,8 & $1: 1$ \\
Básico (0) & 0,6 & 1 & $2: 1$ \\
Máximo(+1) & 0,8 & 1,2 & $3: 1$ \\
\hline
\end{tabular}




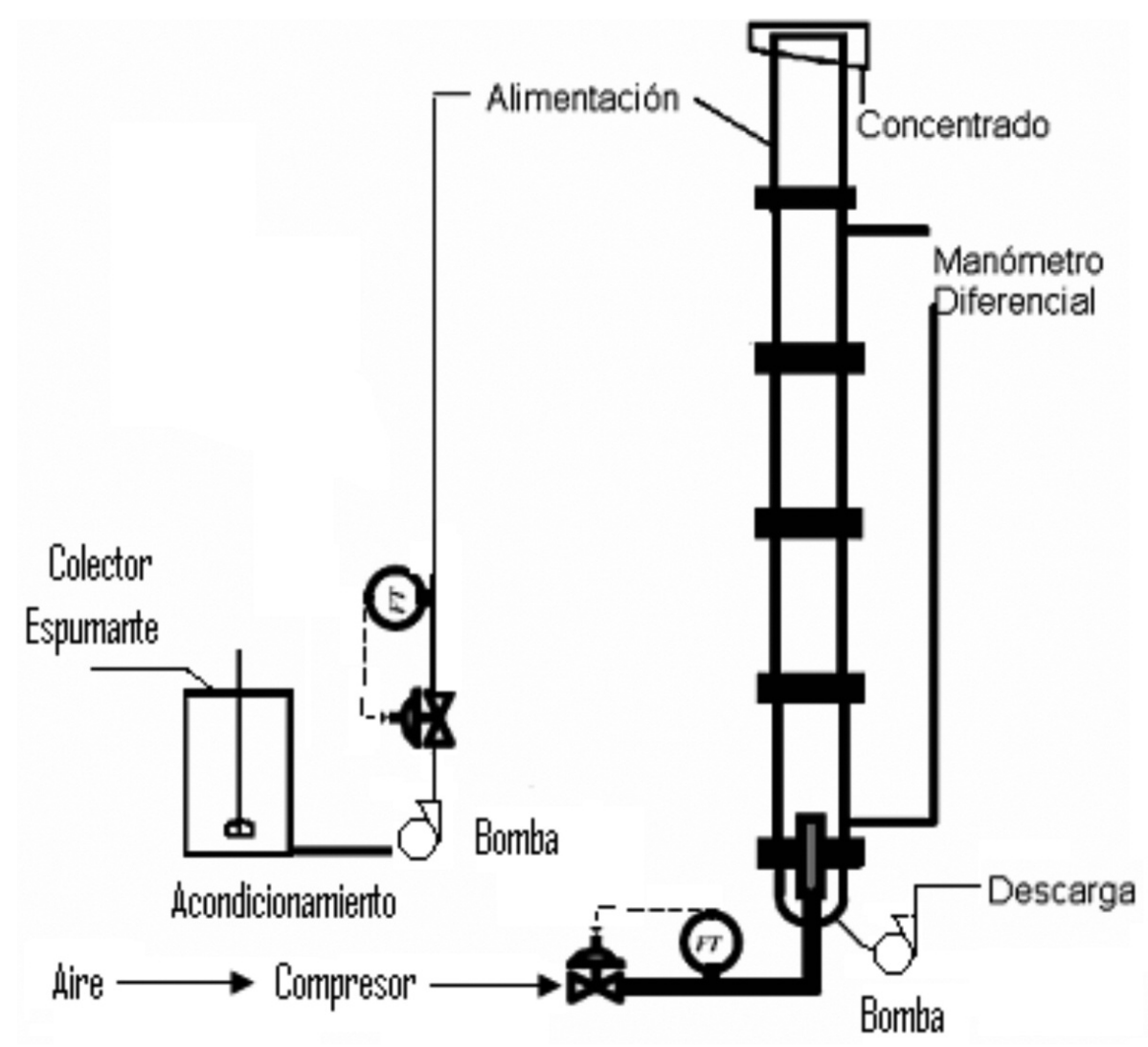

Figura 1. Representación esquemática de la instalación de flotación.

Figure 1. Schematic representation of the flotation installation.

realizó la caracterización de la columna para diferentes concentraciones de espumante, se varían la velocidad superficial del gas y del líquido y se evalúa el comportamiento de los flujos en la columna, la formación de torbellinos y turbulencia en el sistema. La selección del rango de los niveles de estas variables garantiza el régimen laminar en la zona de colección de la

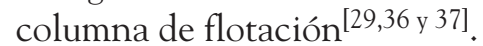

Para el desarrollo del trabajo en un recipiente con agitación constante, se prepararon 901 de solución y se ajustó el pH de trabajo. Posteriormente, se añadió el colector amilxantato de potasio en correspondencia con la relación colector: metal y se homogeneizó durante $10 \mathrm{~min}$, agregando seguidamente el espumante, que se mezcló durante otros $10 \mathrm{~min}$. Seguidamente se llenó la columna de flotación y se fijaron las variables operacionales, flujo de líquido y de gas correspondientes a los valores de velocidad superficial del gas y del líquido, según el diseño de experimentos realizado.

A partir de aquí, se comenzó a suministrar el flujo de aire por la parte inferior de la columna, de acuerdo con el principio a contracorriente, hasta lograr la estabilidad de los flujos; a partir de este instante se separaron los flujos de cola y concentrado y se tomó la primera muestra en éstos y en la alimentación. La descarga de la columna se recircula al tanque de alimentación. Los ensayos se realizaron a temperatura ambiente y durante $1 \mathrm{~h}$ cada uno.

$\mathrm{El} \mathrm{pH}$ de trabajo para el desarrollo de los ensayos correspondientes al diseño de experimentos fue igual a 4,5 y la concentración de cobre, $62 \mathrm{mg} / \mathrm{l}$; estos valores se seleccionaron con el objetivo de simular las características de las aguas residuales de "Mina Grande del cobre", Cuba. Los resultados de las pruebas experimentales se sometieron a una limpieza de datos mediante los criterios de $2 \sigma$ y la $t$ de Student. Con ayuda del paquete Statgraphics Plus 5.0 se realizó un análisis estadístico y se obtiene el modelo matemático-estadístico que caracteriza la colección de cobre mediante la flotación en columna. Este análisis contribuye a establecer las regularidades de este proceso a partir de la identificación del efecto de las variables estudiadas en la recuperación de cobre.

Se realizó además, una serie de 5 experimentos para comprobar el efecto del $\mathrm{pH}$ en la recuperación de cobre durante la flotación con el reactivo colector amilxantato de potasio. Las condiciones operacionales se seleccionaron a partir de los resultados del diseño de experimentos. 


\section{RESULTADOS Y DISCUSIÓN}

\subsection{Flotación en columna de iones cobre con amilxantato de potasio}

Los resultados experimentales indican (Fig. 2) que la recuperación de cobre disminuye con el incremento de la relación colector: metal independientemente del nivel de las variables velocidad superficial del gas y del líquido.

Esto es una consecuencia de la influencia que tiene la fortaleza del enlace colector-metal en la longitud de la cadena carbonada del compuesto.

Se ha demostrado ${ }^{[38]}$ que la diferencia en la capacidad de adsorción de los xantatos, comparada con otros colectores como los carboxilatos, sulfonatos y alquilsulfatos, se debe a que estos compuestos retienen el fuerte carácter hidrofílico aún cuando se haya formado enlace con el metal. En el caso de los xantatos, el enlace tiene fuertes características covalentes, por eso, con longitudes de cadenas carbonadas pequeñas es posible disminuir el carácter hidrofúlico del grupo polar y lograr la flotación del compuesto formado. El incremento de la concentración del colector, fortalece el carácter hidrofílico del grupo polar enlazado al metal. Además, las características propias de los metales de transición de formar complejos acuosos en solución, provoca una disminución de la capacidad de flotación del compuesto formado, debido a presentar mayor tendencia a mantenerse en solución que a adherirse a la burbuja, es decir, predominan las características hidrofílicas sobre las hidrofóbicas.

La disminución de la recuperación con el incremento de la relación colector: metal no es atribuible a la formación de micelas en las condiciones de concentración y temperatura bajo las cuales se realizan los experimentos, donde resulta imposible alcanzar el punto de Krafft ${ }^{[38 \text { y } 39]}$. Además, de acuerdo con las investigaciones realizadas por Stalidis et al. ${ }^{[18]}$,se plantea que los reactivos colectores xantogenados de cadenas cortas se caracterizan por la significativa falta de asociación de la cadena, que explica la incapacidad de la forma aniónica disuelta de formar micelas coloidales. Estas consideraciones fortalecen el hecho de que la naturaleza del metal es determinante.

Para los niveles básico y mínimo de las variables velocidad superficial del líquido y la relación colector: metal según el diseño de experimento, la recuperación de cobre muestra un comportamiento similar con el incremento de la velocidad superficial del gas (Fig. 3); en ambos casos disminuye aunque es más acentuado cuando el nivel de las variables es el mínimo.

Esta conducta se relaciona con el efecto de las corrientes de circulación de las fases continua y dispersa en la columna de flotación, las cuales provocan turbulencias y atentan contra la estabilidad del agre-

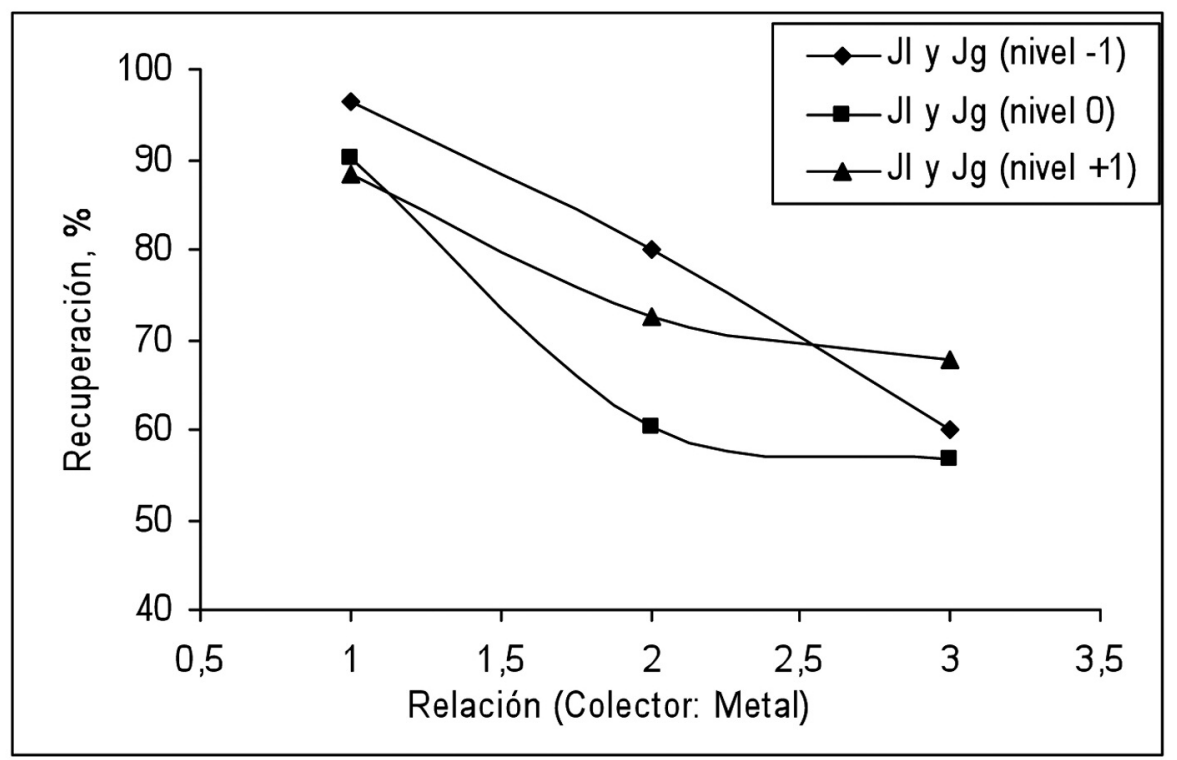

Figura 2. Comportamiento de la recuperación de cobre para niveles iguales de $\mathrm{Jl}$ y Jg, según el diseño experimental, y diferentes relaciones colector: metal.

Figure 2. Behaviour of the copper recovery for similar levels of $\mathrm{Jl}$ and $\mathrm{Jg}$, according to the experimental design, and different collector: metal relations. 


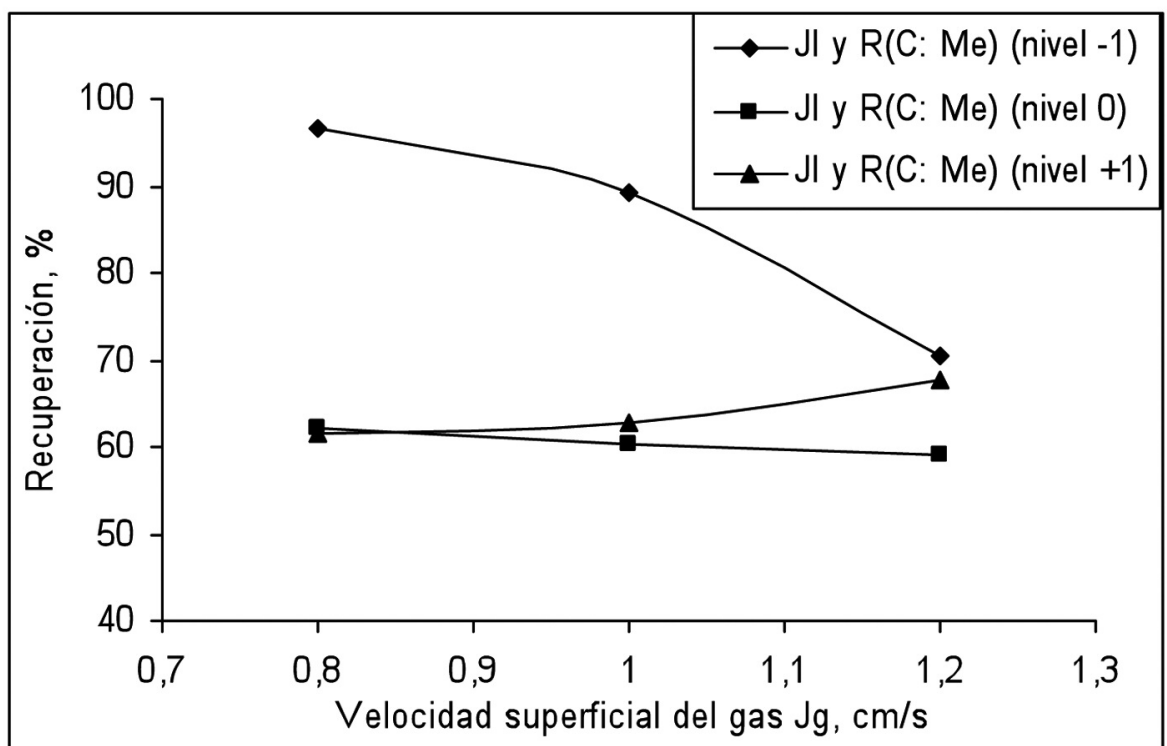

Figura 3. Comportamiento de la recuperación de cobre para niveles iguales de $\mathrm{Jl}$ y relación colector: metal y diferentes valores de Jg.

Figure 3. Behaviour of the copper recovery for similar levels of $\mathrm{Jl}$ and collector: metal relationship and different values of $\mathrm{Jg}$.

gado partícula-burbuja. En la medida que se incrementa la velocidad superficial del gas, siempre que la tensión superficial se mantiene constante, se incrementa el diámetro de la burbuja y se reduce el área superficial disponible para el intercambio de masa ${ }^{[40]}$, condiciones que limitan la recuperación de cobre.
De acuerdo con la figura anterior, con el incremento de la velocidad superficial del gas, cuando el nivel de la relación colector: metal es máximo, se favorece la formación de la especie amildixantato de acuerdo con la siguiente ecuación de reacción, (Ec. (1)):

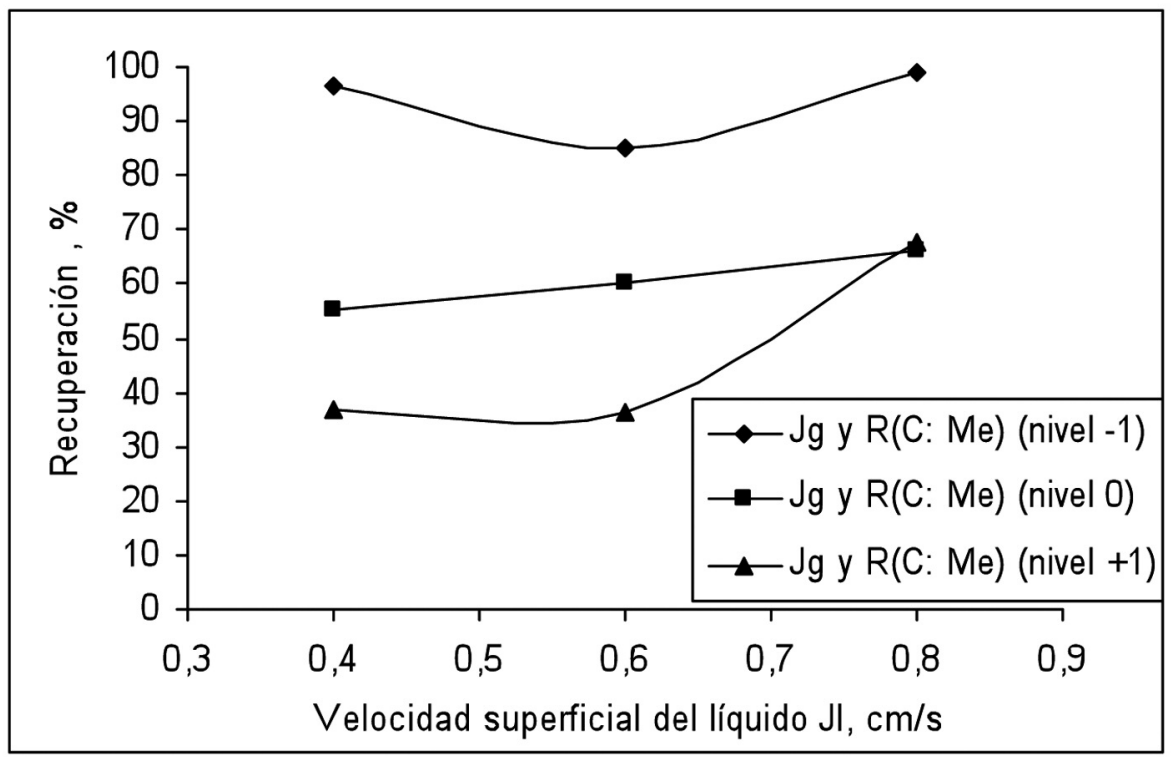

Figura 4. Comportamiento de la recuperación de cobre para niveles iguales de Jg y relación colector: metal y diferentes valores de Jl.

Figure 4. Behaviour of the copper recovery for similar levels of Jg and collector: metal relationship and different values of $\mathrm{Jl}$. 


$$
\begin{gathered}
2 \mathrm{ROCS}_{2}^{-}(\mathrm{ac})+1 / 2 \mathrm{O}_{2}(\mathrm{ac})+\mathrm{H}_{2} \mathrm{O}(\mathrm{ac}) \Leftrightarrow \\
\left(\mathrm{ROCS}_{2}\right)_{2}(\mathrm{ac})+2 \mathrm{OH}^{-}(\mathrm{ac})
\end{gathered}
$$

Esta especie manifiesta propiedades colectoras ${ }^{[41}$ y 42$]$, lo cual puede contribuir con el ligero aumento de la recuperación.

En la figura 4, se puede comprobar el efecto positivo que ejercen en la recuperación las variables velocidad superficial del gas y la relación colector: metal; cuando el nivel de estas variables es mínimo se alcanzan valores superiores al $80 \%$ y el incremento de los mismos trae consigo una notable reducción, lo cual se asocia con la intensificación del mezclado de los flujos de líquido y de gas producto del aumento del diámetro de la burbuja ${ }^{[40]}$.

Cuando se comparan las curvas para los diferentes niveles de Jg y relación colector: metal, inicialmente se refleja una tendencia a la disminución de la recuperación con el incremento de la velocidad superficial del gas. Este comportamiento se corresponde con el fomento del mezclado como consecuencia del incremento del caudal de alimentación y descarga que conduce a la ruptura del agregado partícula-burbuja. Posteriormente se verifica un incremento de la recuperación de cobre, resultado que se relaciona con el ascenso del número de etapas que se desarrolla en función del flujo de la descarga para un tiempo de experimentación de $1 \mathrm{~h}$, según la figura 5 .

La velocidad superficial del líquido es directamente proporcional al flujo de descarga de hundidos, que a su vez es inversamente proporcional al tiempo de residencia. Por tanto para $1 \mathrm{~h}$ de experimentación, el número de etapas aumenta para el procesamiento de los 901 de solución de acuerdo con el tiempo de residencia, que disminuye en función del flujo de colas. A pesar de la disminución del tiempo de retención en la columna por etapa independiente, para igual tiempo de experimentación por ensayo se incrementa el número de etapas. Debe tenerse en cuenta además que el desarrollo del proceso a contracorriente favorece la posibilidad de formación del agregado, especie hidrófoba-burbuja.

Los valores de recuperación promedios para cada condición de experimentación que se excluyen de los gráficos anteriores se relacionan en la tabla II.

Los resultados del análisis de regresión múltiple que se exponen en la tabla III indican que todas las variables de forma independiente y la combinación de la velocidad superficial del líquido y velocidad superficial del gas son estadísticamente significativas.

Tabla II. Recuperación de cobre durante su flotación a partir de soluciones sintéticas

Table II. Copper recovery during their flotation from synthetic solutions

\begin{tabular}{ccccc}
\hline No & $\begin{array}{c}\text { Velocidad } \\
\text { superficial } \\
\text { del líquido } \\
\text { Jl, cm/s }\end{array}$ & $\begin{array}{c}\text { Velocidad } \\
\text { superficial } \\
\text { del gas } \\
\text { Jg, cm/s }\end{array}$ & $\begin{array}{c}\text { Relación } \\
\text { (Colector: } \\
\text { Me) }\end{array}$ & $\begin{array}{c}\text { Recuperación } \\
\text { media } \xi, \%\end{array}$ \\
\hline 1 & -1 & 0 & 1 & 34,31 \\
2 & -1 & 1 & 0 & 47,26 \\
3 & 0 & -1 & 1 & 50,11 \\
4 & 0 & 1 & -1 & 94,91 \\
5 & 1 & -1 & 0 & 65,61 \\
6 & 1 & 0 & -1 & 99,02 \\
\hline
\end{tabular}

El valor 2,09, de la t de Student tabulada, es superior a los valores de t estadístico de los coeficientes para dichas variables.

A partir del análisis matemático-estadístico se obtiene el modelo que caracteriza la flotación de iones cobre, (Ec. (1)), el cual indica la relación de la recuperación de cobre, de las variables velocidad superficial del líquido $\mathrm{Jl},(\mathrm{cm} / \mathrm{s})$, velocidad superficial del gas $\mathrm{Jg},(\mathrm{cm} / \mathrm{s})$, relación colector: metal $\mathrm{R}(\mathrm{C}: \mathrm{Me})$ y sus combinaciones.

$$
\xi(\mathrm{Cu})=68,45+6,28 \cdot \mathrm{Jl}-3,66 \cdot \mathrm{Jg}-
$$

$19,32 \cdot R(C: M e)+8,88 \cdot J l \cdot J g+2,59 \cdot J l \cdot R(C: M e)-$ $2,1 \cdot \mathrm{Jg} \cdot \mathrm{R}(\mathrm{C}: \mathrm{Me})-0,87 \cdot \mathrm{Jl} \cdot \mathrm{Jg} \cdot \mathrm{R}(\mathrm{C}: \mathrm{Me})$ 


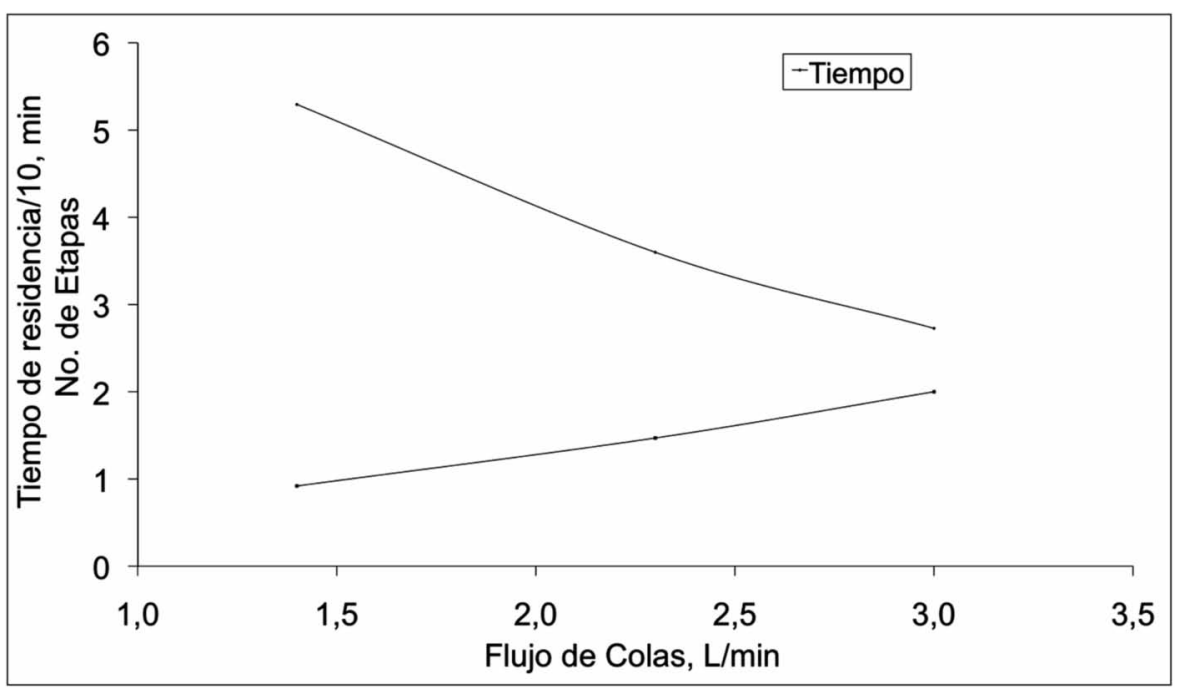

Figura 5. Variación del tiempo de residencia y número de etapas con el flujo de colas.

Figure 5. Variation of the time of residence and number of stages with the flow of tailings.

Tabla III. Resumen del análisis de regresión múltiple

Table III. Multiple regression analysis summaries

\begin{tabular}{lcccc}
\hline \multicolumn{4}{c}{ Variable dependiente: Recuperación , $\boldsymbol{\zeta}(\mathrm{Cu})$} \\
\hline Variables & Estimado & $\begin{array}{c}\text { Error } \\
\text { Standard }\end{array}$ & T Estadístico & Valor P \\
\hline Constante & 68,4474 & 1,43463 & 47,7108 & 0,0000 \\
$\mathrm{Jl}$ & 6,27889 & 1,75706 & 3,57352 & 0,0020 \\
$\mathrm{Jg}$ & $-3,65833$ & 1,75706 & $-2,08208$ & 0,0511 \\
$\mathrm{R}(\mathrm{C}: \mathrm{Me})$ & $-19,3161$ & 1,75706 & $-10,9934$ & 0,0000 \\
$\mathrm{Jl} \cdot \mathrm{Jg}$ & 8,88167 & 2,15195 & 4,12727 & 0,0006 \\
$\mathrm{~J} \cdot \mathrm{R}(\mathrm{C}: \mathrm{Me})$ & 2,58917 & 2,15195 & 1,20317 & 0,2437 \\
$\mathrm{Jg} \cdot \mathrm{R}(\mathrm{C}: \mathrm{Me})$ & $-2,10333$ & 2,15195 & $-0,977409$ & 0,3406 \\
$\mathrm{Jl} \cdot \mathrm{Jg} \cdot \mathrm{R}(\mathrm{C}: \mathrm{Me})$ & 0,87125 & 2,63559 & $-0,330571$ & 0,7446 \\
\hline
\end{tabular}

Según el valor de probabilidad, las combinaciones de las variables relación colector: metal con la velocidad superficial del líquido y del gas, así como la combinación de las tres variables no son estadísticamente significativas para el $90 \%$ de confianza, por lo que se excluyen del modelo.

$$
\begin{gathered}
\xi(\mathrm{Cu})=68,45+6,28 \cdot \mathrm{Jl}-3,66 \cdot \mathrm{Jg}- \\
19,32 \cdot \mathrm{R}(\mathrm{C}: \mathrm{Me})+8,88 \cdot \mathrm{J} \cdot \mathrm{Jg}
\end{gathered}
$$

El valor de $\mathrm{R}^{2}$ indica que el modelo reproduce el $89,23 \%$ de las pruebas experimentales realizadas de acuerdo con el grado de dispersión de los valores de recuperación medio, $\xi$ para cada condición experimental del diseño y el valor del modelo, representado en la figura 6.

Dadas las complejidades que caracterizan el proceso de flotación, donde intervienen variables que se modifican continuamente tales como la composición iónica de la pulpa, la fuerza iónica y la tensión superficial entre otras, este resultado puede considerarse positivo; se destaca además la escala a la cual se desarrolla la investigación y el error absoluto de la investigación igual a 5,7. 


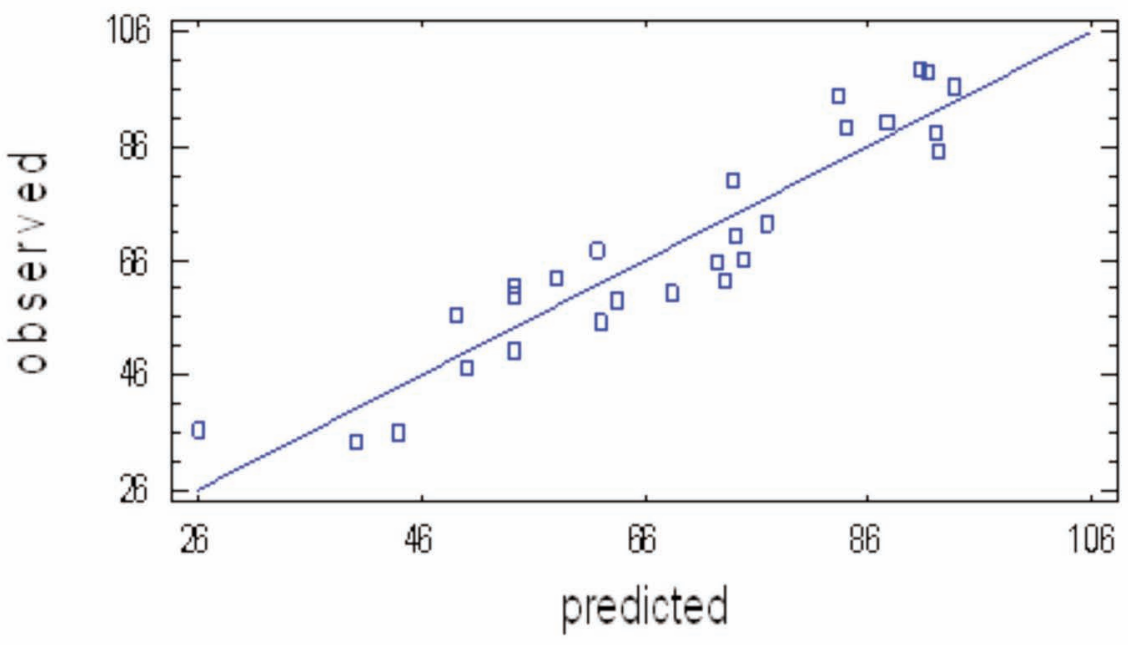

Figura 6. Comportamiento de la recuperación de cobre experimental y predicho.

Figure 6. Behaviour of the experimental and predicted value of copper recovery.

En cuanto al efecto de las variables del modelo, se corrobora el análisis que se realiza con anterioridad donde la velocidad superficial del líquido influye de forma positiva, en cambio las variables velocidad superficial del gas y la relación colector: metal es negativa. Con respecto a la combinación significativa de las variables velocidad superficial del gas y del líquido, su efecto es positivo. Por la magnitud de los coeficientes, la variable de mayor incidencia en los resultados es la relación colector: metal.

\subsection{Efecto del pH en la flotación de complejos amilxantogenados de cobre}

De acuerdo con los resultados según el diseño experimental, se seleccionan las condiciones de las variables independientes bajo las cuales se obtienen los mejores resultados para evaluar el efecto del $\mathrm{pH}$ en la flotación de los complejos amilxantogenados de cobre, donde la relación colector: metal es igual a $1: 1$ y velocidad superficial del líquido y del gas $0,8 \mathrm{~cm} / \mathrm{s}$. Los datos experimentales indican según la figura 7, que la recuperación acumulativa de cobre es superior al $90 \%$ para todos los valores de $\mathrm{pH}$.

Sin embargo, a partir del minuto 50 aproximadamente, se observa una ligera disminución de su valor acumulativo en el experimento a $\mathrm{pH} 12$ con respecto de los $\mathrm{pH}$ inferiores. En estas condiciones tiene lugar la descomposición hidrolítica parcial del ión amilxantato ${ }^{[43]}$ ecuación, de reacción 2, (Ec. (2)):

$$
\begin{aligned}
6 \mathrm{C}_{5} \mathrm{H}_{11} \mathrm{OCS}_{2}(\mathrm{ac})+3 \mathrm{H}_{2} \mathrm{O}=\mathrm{CO}_{3}^{2-}(\mathrm{ac})+3 \mathrm{CS}_{2}(\mathrm{ac}) \\
+2 \mathrm{CS}_{3}^{2-}(\mathrm{ac})+6 \mathrm{C}_{5} \mathrm{H}_{11} \mathrm{OH}(\mathrm{ac})
\end{aligned}
$$

El desarrollo de esta reacción indica que la recuperación a pH 12 disminuye, debido a que parte del ión amilxantato se descompone y disminuye su concentración en solución, por tanto se limita la formación de los complejos de cobre.

Trabajos previos de Ramírez y colaboradores ${ }^{[30 \text { y 40] }}$ realizados a partir del análisis del sistema de reacciones que tiene lugar combinado con el uso de diferentes técnicas analíticas, les permitió dilucidar el mecanismo para la remoción del cobre disuelto por flotación. En ellos se verifica la formación del complejo xantogenado cobre(II), ecuación de reacción 3, y la reducción del cobre(II) en forma de complejo de cobre(I), ecuación de reacción 4; este proceso se verifica en todo el rango de $\mathrm{pH}$, aunque en la medida que este se incrementa la relación de complejos cobre(I)/cobre(II) aumenta. La formación de las especies xantogenadas de cobre producto de la interacción del reactivo colector con los iones cobre, le confiere a los complejos características hidrófobas que posibilitan su remoción durante el proceso de flotación.

$$
\begin{gathered}
2 \mathrm{ROCS}_{2}^{-}(\mathrm{ac})+2 \mathrm{~K}^{+}(\mathrm{ac})+\mathrm{Cu}^{2+}(\mathrm{ac})= \\
2 \mathrm{~K}^{+}(\mathrm{ac})+\mathrm{Cu}\left(\mathrm{ROCS}_{2}\right)_{2}(\mathrm{ac}) \\
2 \mathrm{Cu}\left(\mathrm{ROCS}_{2}\right)_{2}(\mathrm{ac})=\mathrm{Cu}_{2}\left(\mathrm{ROCS}_{2}\right)_{2}(\mathrm{~s})+ \\
\left(\mathrm{ROCS}_{2}\right)_{2}(\mathrm{ac})
\end{gathered}
$$




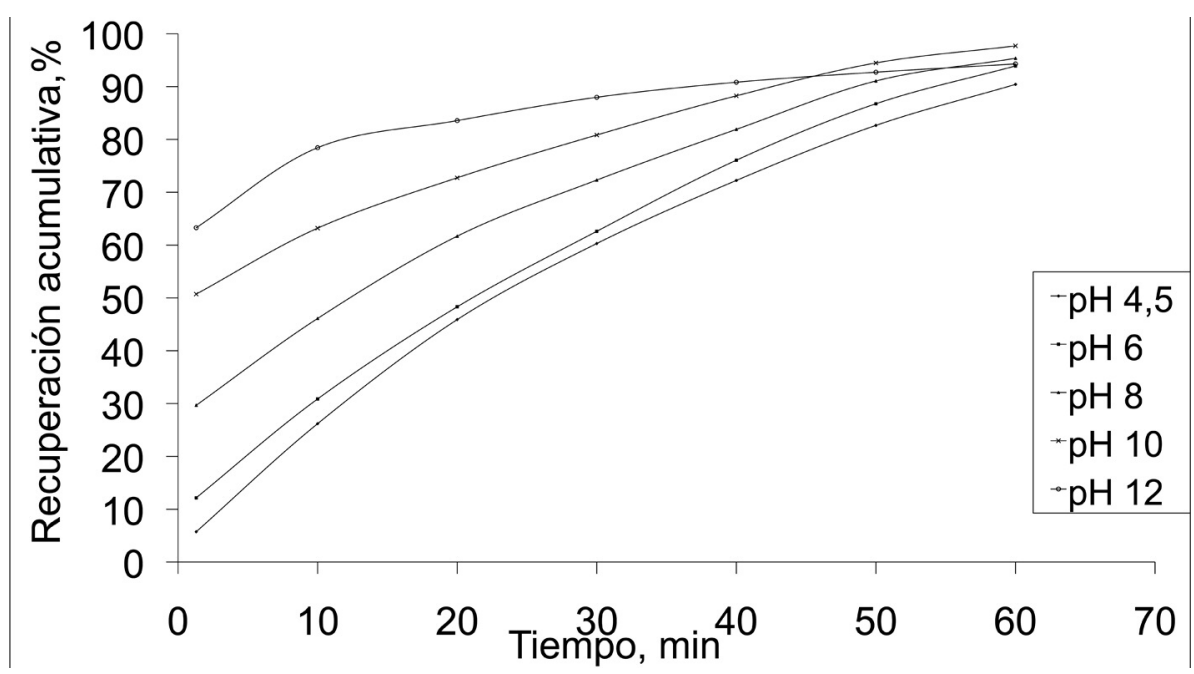

Figura 7. Comportamiento de la cinética de flotación de cobre en función del $\mathrm{pH}$.

Figure 7. Behaviour of the kinetics of copper flotation as a function of $\mathrm{pH}$.

Como se observa en la figura 7, de acuerdo con el planteamiento anterior la cinética del proceso de colección se favorece con el incremento del pH producto de la presencia mayoritaria del precipitado de amilxantato de cobre(I). Por tanto se puede corroborar que el proceso de flotación de cobre disuelto con el reactivo colector amilxantato de potasio se verifica como un proceso mixto de flotación iónica y de flotación de precipitados, aunque se constata un predominio de este último.

\section{CONCLUSIONES}

- Los resultados experimentales demuestran que la flotación de cobre con amilxantato de potasio en columnas de flotación es técnicamente factible. Se establecen las mejores condiciones de las variables relación colector: metal de 1:1, velocidad superficial del líquido y del gas $0,8 \mathrm{~cm} / \mathrm{s}$ para $\mathrm{pH}$ igual a 4,5 .

- La recuperación de especies de cobre se verifica mediante un proceso mixto de flotación iónica y flotación de precipitados, en el rango de $\mathrm{pH}$ de 4 a 13, lo cual indica la potencialidad de los resultados en el tratamiento de soluciones contaminadas con cobre. Se comprueba una fuerte dependencia de las características químicas e hidrodinámicas en la columna de flotación tanto en la cinética de colección como la recuperación.

\section{REFERENCIAS}

[1] S. Babel y T. A. Kurniawan, J. Hazard. Mater. B97 (2003) 219-243.

[2] F. Fu, H. Zeng y Q. Cai, Chemosphere 69 (2007) 1.783-1.789.

[3] J. M. Sun, S. Y. Chang, R. Li y J. C. Huang, Sep. Purif. Technol. 56 (2007) 57-62.

[4] M. A. Barakat, Arabian J. Chem. (2010) 1-17.

[5] T. A. Kurniawan, Y. S. Gilbert, Chan, W.-H. Lo y S. Babel, Chem. Eng. J. 118 (2006) 83-98.

[6] M. Reyes, F. J. Tavera, R. Escudero, F. Patiño, E. Salinas y I. Rivera, Rev. Metal. Madrid 46 (2010) 109-120.

[7] J. Rubio, M. L. Souza y S. W. Smith, Miner. Eng. 15 (2002) 139-155.

[8] A. Mahiroglu, E. Tarlan-Yel y M. F. Sevimli, J. Hazard. Mater. 166 (2009) 782-787.

[9] M. Santander, P. Tapia, O. Pávez, L. Valderrama y D. Guzmán, Rev. Metal. Madrid 45 (2009) 365-374.

[10] F. M. Doyle, Int. J. Miner. Process. 72 (2003) $387-399$.

[11] K. A. Matis y A. I. Zouboulis, Sep. Purif. Methods 36 (2001) 3.777-3.800.

[12] K. A. Matis y P. Mavros, Sep. Purif. Methods 20 (1991) 1- 48.

[13] A. Azareño, P. A. Núñez, E. León, M. Morales, J. Jara y J. L. Rendón, Revista del Instituto de Investigación de la Facultad de Ingeniería Geológica, Minera, Metalúrgica y Geográfica 6 (2003) 82-90.

[14] N. K. Lazaridis, E. N. Peleka, T. D. Karapantsios y K. A. Matis, Hydrometallurgy 74 (2004) 149-156. 
[15] A. A. Abramov y K. S. E. Forssberg, Miner. Process. Ext. Met. Rev 26 (2005) 77- 143.

[16] S. R. Rao, Xanthates and related compounds; 1 ed.; Marcell Dekker: New York, 1971, pp. $1-504$.

[17] G. W. Poling In Flotation-A. M. Gaudin Memorial; Fuerstenau, M. C., Ed.; SME/AIME: New York, 1976, p. 334.

[18] G. A. Stalidis, K. A. Matis y N. K. Lazaridis, Sep. Sci. Technol. 24 (1989) 97 - 109.

[19] R. N. Tipman y J. Leja, Colloid Polym. Sci. 253 (1975) 4-10.

[20] I. Iwasaki y S. R. B. Cooke, J. Am. Chem. Soc. 80 (1958) 285-288.

[21] Z. Sun y W. Forsling, Miner. Eng. 10 (1997) 389-400.

[22] J. Leja, Surface Chemistry of Froth Flotation; Plenum: New York, 1982, pp.1-228.

[23] N. K. Lazaridis, K. A. Matis, G. A. Stalidis y P. Mavros, Sep. Sci. Technol. 27 (1992) 1.743-1.758.

[24] I. Kakovsky In 2nd International Conference on Surface Activity. Butterworths, London, 1957; Vol. 4, pp. 225-237.

[25] J. Simpson, D. Jordán, G. Cifuentes, A. Morales y L. Briones, Rev. Metal 46 (2010) 101-108.

[26] V. A. Ignatkina, V. Samygin, D, y V. A. Bocharov, J. Min. Sci. 45 (2009) 75-79.

[27] P. de Donato, J. M. Cases, M. Kongolo, A. Cartier y J. L. Rivail, Int. J. Miner. Process. 25 (1989) 1-16.

[28] F. J. Tavera, R. Escudero, A. Uribe y J. A. Finch, Afinidad 2000, LVII, pp. 415- 423.

[29] J. A. Finch y G. S. Dobby, Column flotation; 1st ed.; Pergamon Press: Oxford, 1990, pp. $1-179$.

[30] B. Ramírez, J. Tavera y R. Escudero, Min. Geol. 24 (2008) 1-21.
[31] R. Escudero y F. J. Tavera, Trans. Instn Min. Metall. Sec C: Miner Process. Extr. Metall. 111/Proc (2002) 143-148.

[32] S. Shirsat, A. Mandal, G. Kundu y D. Mukherjee, Int. J. Chem. React. Eng. 84 (2003) 38-43.

[33] M. Massinaei, M. Kolahdoozan, M. Noaparast, M. Oliazadeh, J. Yianatos, R. Shamsadini y M. Yarahmadi, Miner. Eng. 22 (2009) 272-278.

[34] A. Uribe S, D. Vazquez V, R. Pérez G y F. Nava A, Miner. Eng. 12 (1999) 937-948.

[35] M. Yahyaei, S. Banisi y H. Javani, Sep. Sci. Technol. (2006) 3.609-3.617.

[36] M. A. Bennett, S. P. Luke, X. Jia, R. W. West y R. A. Williams In 1st World Congress on Industrial Process Tomography; Buxton, Greater Manchester 1999, pp. 54-61.

[37] R. C. Chen, J. Reese y L. S. Fan, AICHE J. 40 (1994) 1.093-1.104.

[38] I. C. Hamilton y R. Woods, Int. J. Miner. Process. 17 (1986) 113-120.

[39] J. S. Duncan, Introduction to colloid \& surface chemistry; 4ta ed.; Butterworth Heinemann: New York, 1992, p. 306.

[40] B. Ramírez, R. Escudero y F. J. Tavera, Min. Geol. 25 (2009) 1.

[41] R. D. Crozier, Flotation: theory, reagents and ore testing; 1 ed.; Pergamon Press plc: Great Britain, 1992, pp.1-343.

[42] R. P. King, Principles of flotation; 1 ed.; South African Institute of Mining and Metallurgy: Johannesburg., 1982, pp. 1-268.

[43] B. Ramírez, R. Escudero, F. Tavera y G. Ruiz In Sohn International Symposium "Advanced processing of metals and materials"; TMS (The Minerals, Metals \&Materials Society: San Diego, 2006; Vol. 3 Thermo and physicochemical principles: special materials and aqueous and electrochemical processing, p. 547. 